\title{
Associations between bone attenuation and prevalent vertebral fractures on chest CT scans differ with vertebral fracture locations
}

\author{
J.H.M. Driessen ${ }^{1,2,3}$ • M.J. van Dort ${ }^{1}$ (D) - E.A.P.M. Romme ${ }^{4}$ - E.F.M. Wouters ${ }^{5}$ • F.W.J.M. Smeenk ${ }^{6,7} \cdot$ B. van Rietbergen ${ }^{8}$ • \\ J.P.W. van den Bergh ${ }^{1,9,10}$ • P. Geusens ${ }^{9}$
}

Received: 8 March 2020 / Accepted: 27 October 2020 / Published online: 16 February 2021

(C) The Author(s) 2021

\begin{abstract}
Summary Vertebral fracture (VF) locations are bimodally distributed in the spine. The association between VF and bone attenuation (BA) measured on chest CT scans varied according to the location of VFs, indicating that other factors than only BA play a role in the bimodal distribution of VFs.

Introduction Vertebral fractures (VFs) are associated with low bone mineral density but are not equally distributed throughout the spine and occur most commonly at T7-T8 and T11-T12 ("cVFs") and less commonly at T4-T6 and T9-T10 ("lcVF"). We aimed to determine whether associations between bone attenuation (BA) and VFs vary between subjects with cVFs only, with lcVFs only and with both cVFs and lcVFs.

Methods Chest CT images of T4-T12 in 1237 smokers with and without COPD were analysed for prevalent VFs according to the method described by Genant (11,133 vertebrae). BA (expressed in Hounsfield units) was measured in all non-fractured vertebrae (available for 10,489 vertebrae). Linear regression was used to compare mean BA, and logistic regression was used to estimate the association of BA with prevalent VFs (adjusted for age and sex).

Results On vertebral level, the proportion of cVFs was significantly higher than of lcVF (5.6\% vs $2.0 \%)$. Compared to subjects without VFs, BA was $15 \%$ lower in subjects with cVFs $(p<0.0001), 25 \%$ lower in subjects with lcVFs $(p<0.0001)$ and lowest in subjects with cVFs and lcVFs $(-32 \%, p<0.0001)$. The highest ORs for presence of VFs per -1 SD BA per vertebra were found in subjects with both cVFs and lcVFs (3.8 to 4.6).

Conclusions The association between VFs and BA differed according to VF location. ORs increased from subjects with cVFs to subjects with lcVFs and were highest in subjects with cVFs and lcVFs, indicating that other factors than only BA play a role in the bimodal VF distribution.
\end{abstract}

Trial registration Clinicaltrials.gov identifier: NCT00292552

Keywords Bone attenuation · CT scans · Vertebral fractures

M.J. van Dort

m.vandort@maastrichtuniversity.nl

1 Department of Internal Medicine, NUTRIM School of Nutrition and Translational Research in Metabolism, Maastricht University Medical Centre+ (MUMC+), Postbus 5800, 6202, AZ Maastricht, The Netherlands

2 Cardiovascular Research Institute Maastricht, Maastricht University Medical Centre+ (MUMC+), Postbus 5800, 6202, AZ Maastricht, The Netherlands

3 Department of Clinical Pharmacy and Toxicology, Maastricht University Medical Centre+ (MUMC+), Postbus 5800, 6202, AZ Maastricht, The Netherlands

4 Department of Respiratory Medicine, Rijnstate Hospital, Postbus 9555, 6800, TA Arnhem, The Netherlands
5 Department of Respiratory Diseases, Maastricht University Medical Centre+ (MUMC+), Postbus 5800, 6202, AZ

Maastricht, The Netherlands

6 Department of Respiratory Medicine, Catharina Hospital, Postbus 1350, 5602, ZA Eindhoven, The Netherlands

7 School of Health Professions Education, Faculty of Health Medicine and Life Sciences, Maastricht University Medical Centre+ (MUMC+), Postbus 5800, 6202, AZ Maastricht, The Netherlands

8 Faculty of Biomedical Engineering, Section Orthopaedic Biomechanics, Eindhoven University of Technology, Postbus 513, 5600, MB Eindhoven, The Netherlands

9 Department of Internal Medicine/Rheumatology, Maastricht University Medical Centre+ (MUMC+), Postbus 5800, 6202, AZ Maastricht, The Netherlands

10 Department of Internal Medicine, VieCuri Medical Centre, Venlo, The Netherlands 


\section{Introduction}

Vertebral fractures (VFs) are the most common fractures in the population older than 50 years [1]. Subjects with prevalent VFs have lower bone mineral density (BMD) in the lumbar spine and hip than subjects without VFs [2], and low BMD in the lumbar spine and femoral neck is a risk factor for incident VFs [3].

However, the prevalence of VFs is not equally distributed across the spine [4], and in the thoracic spine, it is highest at T7-T8 and T11-T12 [5-7]. The reasons for this bimodal distribution of VFs remain unclear. Two hypotheses have been explored in this context, one exploring the association between BMD and the location of VFs and one exploring the association between vertebral loading and the location of VFs.

In several studies the associations between BMD and the presence or incidence of VFs were evaluated according to their locations. In a community-based study, prevalent VFs in the upper spine were more strongly associated with BMD (measured by QCT in T10 and L3) than VFs in the lower spine [8]. In the fracture intervention trial, each SD decrease in the lumbar spine was associated with 2.1 times greater odds of new VFs in the upper spine (T4-T10) compared with 1.5 times for lower spine VFs (T11-L4) with a statistical difference between the two ratios [7]. In the VERO trial, patients with only prevalent VFs in other spine regions than T12 and L1 had significantly lower BMD (measured by DXA in the spine, the femoral neck and total hip) than patients with only T12 and/or L1 VFs [9]. The authors of these studies concluded that VFs in the upper spine are more related to bone fragility than VFs in the lower spine and that other factors than BMD play a role in the unequal distribution of VFs in the spine [7-9].

A study on the biomechanical loading of vertebrae showed that the thoracic regions with the highest prevalence of VFs (T7-T8 and T11-T12) are also the thoracic regions that are at highest compression load during daily activities such as bending and lifting objects [10-13]. A study on the epidemiology of traumatic vertebral fractures indicated that the vertebrae T11-T12 are at highest risk for fracture when falling [14]. These findings provided a biomechanical mechanism for the higher incidence of fractures in these regions compared to other spinal regions $[10,13]$.

None of the above-mentioned studies evaluated the association between BMD and VF location separately in subjects with VFs at the most common levels and subjects with VFs at the less common vertebral levels.

Previously taken clinical computed tomography $(\mathrm{CT})$ scans of the chest performed in the context of lung diseases can be used for opportunistic screening for the presence of VFs and to measure BMD in the vertebrae by bone attenuation [15-18]. For this study, we used the chest CT scans of the ECLIPSE study, a large cohort of current and former smokers with and without COPD [19].

\section{Aims}

The purpose of this study was to determine whether associations between bone attenuation (BA) and prevalent VFs vary between subjects with VFs at more common locations T7-T8 and T11-T12 (cVFs) versus less common locations (lcVFs).

We hypothesized that BA is higher in subjects with cVFs than in subjects with lcVFs, because other factors such as a sudden load during a fall or more strenuous daily activities might contribute to a higher extent in VFs at common locations.

\section{Methods}

\section{Subjects}

We included subjects from the ECLIPSE study (Evaluation of COPD Longitudinally to Identify Predictive Surrogate Endpoints; Clinicaltrials.gov identifier NCT00292552; GlaxoSmithKline study SCO104960). Detailed inclusion and exclusion criteria were described elsewhere [19-21]. Current or former smokers (40-75 years old) with moderate to very severe COPD (stage II-IV according to the Global Initiative for Chronic Obstructive Lung Disease guidelines [22]), or without COPD, with a smoking history of at least 10 pack years were included.

\section{Measurements}

\section{Chest CT scans}

CT scans of the chest were performed at full inspiration (120 kV peak, $40 \mathrm{mAs}, 1.00$ or 1.25 -mm volumetric acquisition, General Electric [GE] or Siemens), as described earlier [17]. CT scanners were calibrated regularly using industry and institutional standards.

Of all sagittal reformats containing the spine, the contrast was adjusted to (partly) eliminate soft tissue. Subsequently, all sagittal reformats containing the spine were superposed to create simulated lateral X-ray 2D images using MATLAB (version R2013a, MathWorks, Natick, MA, USA). Images were exported in DICOM-format.

\section{Vertebral fracture assessment}

Detailed information has been reported elsewhere [17]. Briefly, VFs in vertebrae T4-T12 were first semiquantitatively assessed, where vertebrae were graded as deformed or not deformed. Vertebrae with deformations due to qualitative features of morphology such as Schmorl's nodes, Scheuermann's disease, platyspondyly or fusion of vertebrae were excluded. In case of height loss in the vertebral body at 
the anterior side, in the middle or in the total vertebral body without other deformities, vertebrae were subsequently morphometrically assessed using the SpineAnalyzer software (Optasia Medical, Cheadle, UK [18, 23, 24]). VF severity was classified according to the method described by Genant et al. (grade 1, 20-25\% height reduction; grade 2, 25-40\%; grade $3,>40 \%$ ) [25].

Subjects were classified according to the presence and location of their VFs. Four subgroups were created: subjects with no prevalent VFs, subjects with VFs only at common locations (cVFs: T7-T8, T11-T12), subjects with VFs only at less common locations (lcVFs: T4-T6, T9-T10) and subjects with VFs at both common and less common locations combined.

\section{Bone attenuation}

In a previous study, Romme et al. showed that BA measurements on CT correlated well with BMD measurements on DXA in a COPD population $(r=0.827, p<0.001)$ [16]. BA was measured on $\mathrm{CT}$ in vertebrae $\mathrm{T} 4$ to $\mathrm{T} 12$, using a selfwritten algorithm in MATLAB (R2013a, MathWorks, Natick, MA, USA). In a substudy of 25 subjects, ICC's of triple measurements of the same image acquisition showed excellent agreement $($ ICC $=0.998$ [0.996-0.999]; single measures, two-way random, absolute agreement, data not published). Fractured vertebrae were excluded from BA measurements, because their BMD is increased following fracture healing due to callus formation. BA was measured as the mean of T4 to T12 and expressed in Hounsfield units (HU). Analyses were performed using the mean BA of all nonfractured vertebrae ("total BA"). Because there is a gradual decreases in BMD from T4 to L3, with Pearson's correlations of $>0.90$ between thoracic and lumbar vertebrae [26], we also used the mean BA per vertebra ("local BA").

More details about subjects, image processing, VF assessment and BA measurement have been published elsewhere [27].

\section{Statistics}

Baseline characteristics were compared between the different fracture groups using ANOVA for continuous variables and chi-square test for categorical data. The proportion of VFs at common locations versus less common locations was compared with a McNemar's test for dependent proportions.

Linear regression (proc reg) was used to compare total spine BA and BA per vertebra between the different groups (no VF, only cVF, only lcVF and both $\mathrm{cVF}$ and lcVF). The measured BA was the dependent variable, and the different fracture groups were used as independent variables as well as age and sex. Logistic regression (proc logistic) was used to estimate the OR per $50 \mathrm{HU}$ (approximately $1 \mathrm{SD}$ ) lower BA and the risk of any VF, a cVF, a lcVF and both cVFs and lcVFs. For this analysis, the event of interest (any VF, a
$\mathrm{cVF}$, a lcVF or both cVFs and lcVFs) was the dependent variable and $\mathrm{BA} / 50$ was the independent variable. Age and sex were also added as independent variables.

Based on loads on the vertebrae from the manuscript by Bruno et al. [10], the load/BA ratio (also referred as "phi"; the ratio of the applied impact force to the bone strength [28]) was indirectly calculated.

Analyses were performed using SAS 9.4 (SAS Institute, Cary, NC, USA). Figures were created using Microsoft Excel 2010.

\section{Results}

Baseline characteristics of the 1237 subjects are given in Table 1 . Mean age of subjects was 61 years, $61 \%$ were men, $81 \%$ had COPD, $42 \%$ were current smokers and $58 \%$ were former smokers. Presence of VFs could be measured in 11,055 vertebrae, and BA was available for 10,489 nonfractured vertebrae.

Prevalent VFs were most frequent in T7-T8 ( $>6 \%$ of vertebrae) and T11-T12 (> 4\% of vertebrae) (Fig. 1a). Similar bimodal patterns were found for prevalent grades 1, 2 and 3 VFs separately (Fig. 1b). The proportion of VFs at common locations was $5.6 \%$ compared to $2.0 \%$ at the less common locations $(p<0.0001)$.

Of the 1237 subjects, 239 (19\%) had at least one VF, $197(16 \%)$ had at least one cVF, $100(8 \%)$ at least one lcVFs, $139(11 \%)$ had only cVFs, 42 (3\%) had only lcVFs and $58(5 \%)$ had both.

\section{Total BA in subjects according to VF locations}

Compared to subjects without a VF and adjusted for age and sex, total BA was $21 \%$ lower in subjects with a prevalent VF (128 \pm 43 versus $162 \pm 46 \mathrm{HU}$, mean difference 34HU, $p<0.0001$ ).

Total BA was $138 \pm 44 \mathrm{HU}$ in subjects with only cVFs (mean difference without VFs: $23 \mathrm{HU}), 122 \pm 35 \mathrm{HU}$ in subjects with only lcVFS $(p<0.0001$ versus cVFs, mean difference without VFs: $40 \mathrm{HU}$ ) and $110 \pm 36 \mathrm{HU}$ in subjects with a combination of cVFs and lcVFs $(p<0.0001 \mathrm{vs} \mathrm{cVFs}$, mean difference without VFs: $52 \mathrm{HU}$ ) (Fig. 2). After adjustment for age and sex, all these total BA values were significantly $(p<0.0001)$ lower compared to subjects without a VF. The gradual trend in decrease in total BA between no VFs, only cVFs, only lcVFs and cVFs and lcVFs combined was significant $(p<0.0001)$.

\section{Local BA per vertebra according to VFs locations}

BA gradually decreased from $\mathrm{T} 4$ to $\mathrm{T} 12$, for both subjects without as well as subjects with at least one VF (Fig. 3a). Therefore, we additionally analysed local BA in each individual non-fractured vertebra. Local BA was at any level 
Table 1 Baseline characteristics

\begin{tabular}{|c|c|c|c|c|c|c|c|c|c|c|c|}
\hline \multirow[b]{3}{*}{ Age (mean, SD) } & \multirow{2}{*}{\multicolumn{2}{|c|}{$\begin{array}{l}\text { All patients } \\
N=1237\end{array}$}} & \multirow{2}{*}{\multicolumn{2}{|c|}{$\begin{array}{l}\text { No VF } \\
N=998\end{array}$}} & \multirow{2}{*}{\multicolumn{2}{|c|}{$\begin{array}{l}\text { Only common } \\
\text { location }\end{array}$}} & \multirow{2}{*}{\multicolumn{2}{|c|}{$\begin{array}{l}\text { Only less } \\
\text { common location }\end{array}$}} & \multicolumn{2}{|c|}{ Both locations } & \multirow{3}{*}{$\begin{array}{c}p \text { value }^{\mathrm{a}} \\
<0.0001\end{array}$} \\
\hline & & & & & & & & & \multicolumn{2}{|c|}{$N=58$} & \\
\hline & 61.3 & 8.0 & 60.7 & 8.0 & 63.6 & 7.3 & 63.8 & 7.0 & 64.9 & 7.1 & \\
\hline Men $(\mathrm{N}, \%)$ & 756 & 61.1 & 582 & 58.3 & 97 & 69.8 & 34 & 81.0 & 43 & 74.1 & 0.0003 \\
\hline BMI (mean, SD) & 25.8 & 4.5 & 25.8 & 4.5 & 26.2 & 4.7 & 25.6 & 4.8 & 24.2 & 4.0 & 0.04 \\
\hline Height (mean, SD) & 170.1 & 9.1 & 169.8 & 9.1 & 171.4 & 8.9 & 171.4 & 7.5 & 171.0 & 8.7 & 0.12 \\
\hline Weight (mean, SD) & 74.8 & 15.8 & 74.6 & 15.6 & 77.5 & 17.1 & 75.4 & 15.1 & 71.3 & 14.7 & 0.07 \\
\hline $\operatorname{COPD}(N, \%)$ & 997 & 80.6 & 795 & 79.7 & 118 & 84.9 & 32 & 76.2 & 52 & 89.7 & 0.12 \\
\hline Former smoker $(N, \%)$ & 713 & 57.6 & 560 & 56.1 & 90 & 64.7 & 30 & 71.4 & 33 & 56.9 & 0.07 \\
\hline Current smoker $(N, \%)$ & 524 & 42.4 & 438 & 43.9 & 49 & 35.3 & 12 & 28.6 & 25 & 43.1 & \\
\hline Pack years (mean, SD) & 43.3 & 24.8 & 42.3 & 23.6 & 46.2 & 29.1 & 50.8 & 30.6 & 47.5 & 27.6 & 0.03 \\
\hline Sum vertebral fractures $(\mathrm{T} 4-\mathrm{T} 12: N, \%)$ & & & & & & & & & & & $<0.0001$ \\
\hline 0 & 998 & 80.7 & 998 & 100.0 & & & & & & & \\
\hline 1 & 139 & 11.2 & & & 104 & 74.8 & 35 & 83.3 & & & \\
\hline 2 & 63 & 5.1 & & & 32 & 23.0 & 7 & 16.7 & 24 & 41.4 & \\
\hline$>2$ & 37 & 3.0 & & & 3 & 2.2 & & & 34 & 58.6 & \\
\hline BA (mean, SD) & 155.6 & 47.5 & 162 & 46.3 & 138 & 44.3 & 122 & 35.1 & 110 & 36.1 & $<0.0001$ \\
\hline
\end{tabular}

Common locations, T7, T8, T11, T12; less common locations, T4-T7, T9, T10

Abbreviations: $V F$ vertebral fracture, $S D$ standard deviation, $B M I$ body mass index, $C O P D$ chronic obstructive pulmonary disease, $B A$ bone attenuation a) Differences between groups were assessed using ANOVA for continuous variables and chi-square test for categorical data
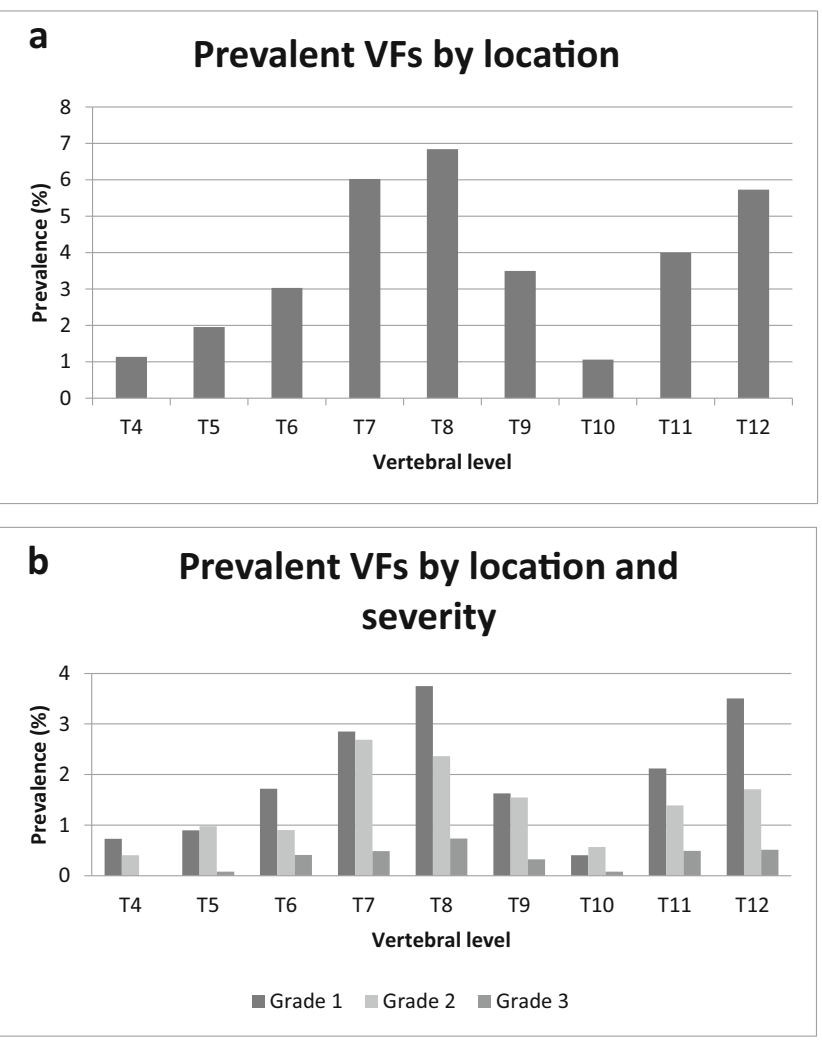

Fig. 1 Proportion of prevalent VFs (a) and prevalent VFs according to severity (b) at vertebrae T4-T12 on chest CT scans. Abbreviations: VF = vertebral fracture significantly lower in the group of subjects with at least one prevalent VF compared to subjects without prevalent VFs, after adjustment for age and sex (Fig. 3a).

In Fig. 3b, local BA is shown at each vertebral level for subjects without prevalent VFs, with only cVFs, only lcVFs or both cVFs and lcVF.

Linear regression analysis showed that, after adjustment for age and sex, at each vertebral level, local BA was significantly lower comparing cVFs, lcVFs and both cVFs and lcVFs to the no VF group. At T4, T5 and T6, there was a significant difference in BA between the only lcVF group and the only cVF group. The local BA of the $\mathrm{cVF}$ and lcVF combined group was significantly lower at each location, except T8, as compared to the local BA of the only $\mathrm{cVF}$ group.

In Table 2, the ORs (adjusted for age and sex) for having any prevalent $\mathrm{VF}$, only cVFs, only lcVFs or combined per50 HU BA (approximately one standard deviation) are shown.

Each $50 \mathrm{HU}$ decrease of BA was associated with a 2.2-3.4 times greater odds of having a lcVFs. These ORs were higher than the odds of a cVFs (OR: 1.5-1.9) and were the highest for combined lcVFs and cVFs.

\section{Load/BA ratio}

In subjects with VFs, the calculated load/BA ratio was higher $34 \%$ than in subjects without VFs $(p<0.0001)$. In 
Fig. 2 Comparison of total BA between subjects without a VF and subjects with VFs according to the regions of VF locations. Differences between groups were adjusted for age and sex. There was a significant trend in the gradual lower BA from no VFs towards $\mathrm{cVFs}$ and lcVFs combined. Abbreviations: BA, bone attenuation; VF, vertebral fracture; $\mathrm{cVF}$, prevalent vertebral fracture at common location (T7T8, T11-T12); lcVF, prevalent vertebral fracture at less common location (T4-T6, T9-T10)

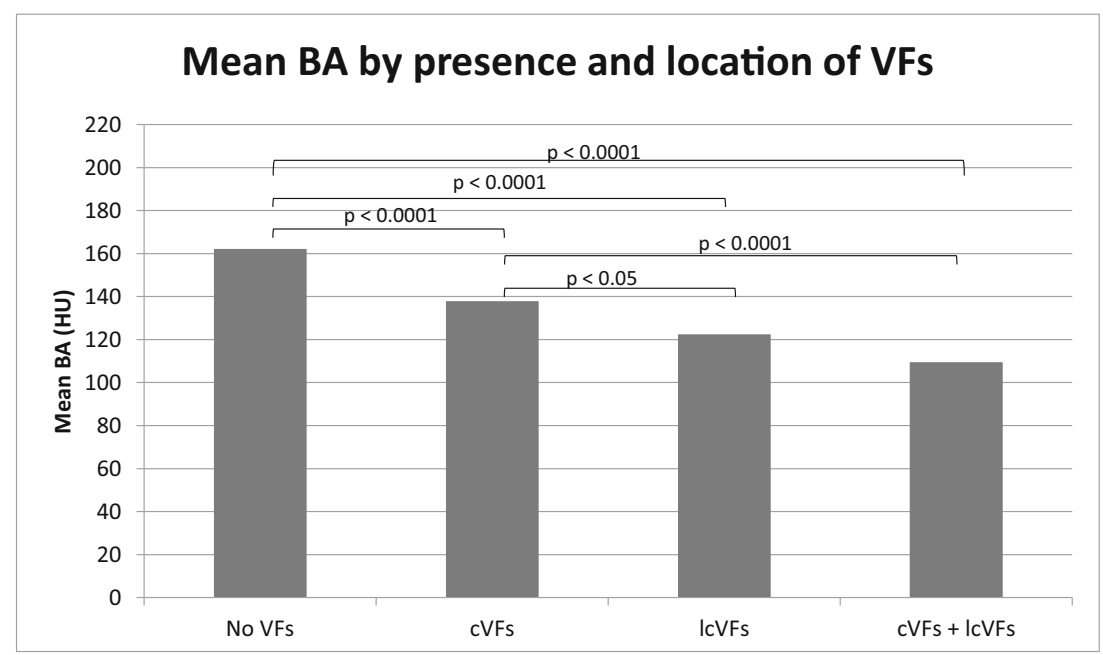

subjects with only cVFs (the most frequent and most loaded regions with a decreased $\mathrm{BA}$ of $15 \%$ ), the load/BA ratio was significantly higher $(+25 \%, p<0.0001)$ than in subjects without VFs. In subjects with both cVFs and lcVFs (having a $32 \%$ lower BA), the load/BA ratio was significantly higher $(+63 \%, p<0.001)$ than in subjects without VFs. Thus, cVFs occurred in subjects with a higher BA and a lower load/BA ratio than in subjects with both cVFs and lcVFs.
Fig. 3 a Mean (with standard error of the mean) local bone attenuation stratified by the presence of vertebral fractures and $\mathbf{b}$ mean (with standard error of the mean) local bone attenuation stratified by the presence and location of vertebral fractures. Abbreviations: VF, vertebral fracture; $\mathrm{HU}$,

Hounsfield units; cVF, prevalent vertebral fracture at common location (T7-T8, T11-T12); $\mathrm{lcVF}$, prevalent vertebral fracture at less common location (T4-T6, T9-T10)

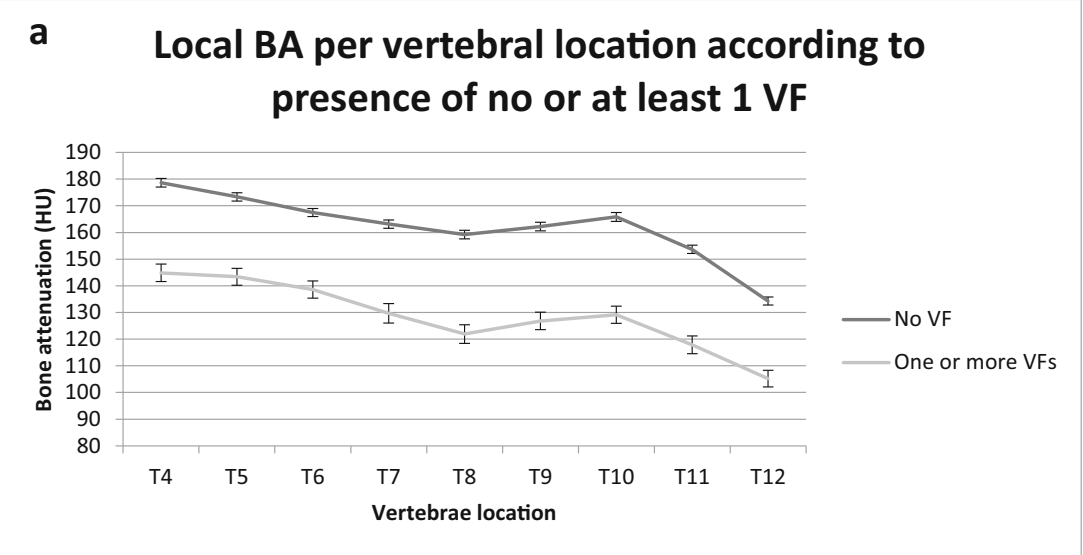

b Local BA stratified by presence and location of VFs

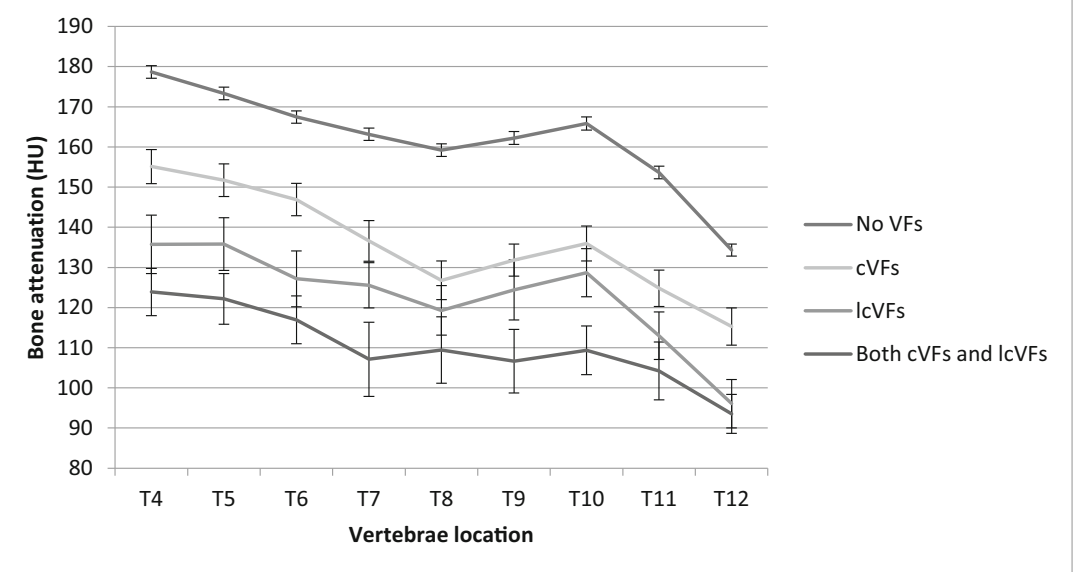


Table 2 Odds ratios for the presence of a VF per - $50 \mathrm{HU}$ in bone attenuation, stratified for VF locations

\begin{tabular}{lllll}
\hline & \multicolumn{1}{l}{ Outcome } & & \\
\cline { 2 - 5 } BA measured at location & $\begin{array}{l}\text { At least one VF at any location } \\
\text { OR }(95 \% \mathrm{CI})^{\mathrm{a}}\end{array}$ & $\begin{array}{l}\text { At least one cVF } \\
\text { OR }(95 \% \mathrm{CI})^{\mathrm{a}}\end{array}$ & $\begin{array}{l}\text { At least one lcVF } \\
\text { OR }(95 \% \mathrm{CI})^{\mathrm{a}}\end{array}$ & $\begin{array}{l}\text { At least one cVF and one lcVF } \\
\text { OR }(95 \% \mathrm{CI})^{\mathrm{a}}\end{array}$ \\
\hline T4 & $2.13(1.77-2.55)$ & $1.59(1.27-1.99)$ & $2.87(1.94-4.24)$ & $3.84(2.65-5.56)$ \\
T5 & $2.08(1.71-2.51)$ & $1.51(1.20-1.90)$ & $2.79(1.85-4.20)$ & $4.30(2.84-6.52)$ \\
T6 & $2.02(1.65-2.46)$ & $1.43(1.14-1.80)$ & $3.35(2.03-5.54)$ & $4.20(2.76-6.41)$ \\
T7 & $2.19(1.76-2.72)$ & $1.62(1.23-2.13)$ & $2.74(1.87-4.02)$ & $4.03(2.45-6.62)$ \\
T8 & $2.49(1.97-3.14)$ & $1.93(1.41-2.63)$ & $2.84(1.93-4.18)$ & $3.94(2.40-6.49)$ \\
T9 & $2.21(1.81-2.70)$ & $1.78(1.40-2.26)$ & $2.38(1.56-3.61)$ & $4.57(2.91-7.19)$ \\
T10 & $2.12(1.77-2.56)$ & $1.63(1.30-2.04)$ & $2.21(1.53-3.20)$ & $4.43(3.00-6.57)$ \\
T11 & $2.42(1.94-3.02)$ & $1.79(1.35-2.37)$ & $2.86(1.90-4.30)$ & $4.40(2.81-6.87)$ \\
T12 & $2.24(1.74-2.87)$ & $1.49(1.09-2.06)$ & $3.20(1.98-5.17)$ & $3.86(2.36-6.32)$ \\
\hline
\end{tabular}

${ }^{\text {a }}$ Adjusted for age and sex

Abbreviations: $V F$ vertebral fracture, $H U$ Hounsfield units, $O R$ odds ratio, $C I$ confidence interval, $c V F$ prevalent vertebral fracture at common location (T7-T8, T11-T12), lcVF prevalent vertebral fracture at less common location (T4-T6, T9-T10)

\section{Discussion}

Our data showed that total BA in the thoracic spine was significantly lower in subjects with at least one VF. In addition, total BA was significantly lower in subjects with only lcVFs compared to subjects with only cVFs. The odds for a prevalent $\mathrm{VF}$ per decrease of $50 \mathrm{HU}$ in BA varied according to $\mathrm{VF}$ location with the lowest OR for $\mathrm{cVFs}$, higher ORs for lcVFs and highest ORs for cVFs and lcVFs combined.

The bimodal distribution of prevalent VFs with clustering in two peaks (T7-T8 and T11-T12) was similar as in a Dutch population-based cohort study based on a random sample and the Fracture Intervention Trial in the USA [7, 29].

Subjects with VFs had lower total and local BA than subjects without a VF. This is in line with the finding that subjects with VFs have lower BMD in the lumbar spine or hip $[2,30]$ and that prevalent VFs are associated with micro-architectural deterioration in the distal radius and tibia measured by HRpQCT [31]. This indicates that prevalent VFs are associated with generalized bone fragility throughout the axial and peripheral skeleton.

Our main finding was that the associations between BA and VFs differed according to the location of VFs. Previous studies have shown that associations between BMD and VFs differ according to VF locations. Two studies reported a stronger association between BMD and upper spine VFS than with lower spine VFs [7, 8]. One study reported a lower BMD in subjects with only VFs outside T12-L1 compared to subjects with only VFs in T12-L1 [9]. However, these studies did not report the associations between BMD and VFs according to both locations with the highest prevalence of VFs. Our results indicate that lcVFs occur in subjects with a more fragile thoracic spine than subjects with the most prevalent cVFs. This is further supported by the higher ORs for the presence of VFs per one SD lower BA per vertebra in subjects with only lcVF than in subjects with only cVFs.

Thus, the degree of vertebral bone fragility varies between subjects according to the thoracic spine locations of VFs. The heterogenous structural failure throughout the thoracic spine can thus not be explained by BA. The most frequent VFs are found in subjects with a higher BA than in subjects with lcVFs. As with any bone that fractures, vertebrae are likely to fracture when the load imposed on the bone exceeds the bone strength [32]. However, the load on vertebrae differs according to their location.

In a retrospective study of 562 patients with a traumatic fracture of the spine, 219 (39\%) had occurred after a fall, predominantly at T11 (4\%), T12 (14\%) and L1 (29\%) [14]. Thus, in the presence of a clear acute trauma such as a fall, VFs occur by preference at the thoracolumbar junction and less in other spine regions. This cannot be explained by bone fragility, as subjects with only T11-T12 VFs in the VERO trial had higher BMD (in spine and hip) than patients with VFs at other locations, and subjects with these VFs in our study had higher BA than subjects with lcVFs [9].

Remarkably, however, and in contrast to non-vertebral fractures, most VFs do not occur after a fall or overt trauma and do not present with the acute clinical signs and symptoms of a fracture. Such VFs are reported as subclinical [33], spontaneous [13], a traumatic or non-traumatic [34] or are detected incidentally in population surveys [29].

The question then arises which other factors than a fall or a severe trauma could explain the presence of subclinical VFs and its bimodal distribution over the spine. An interesting observation is that the load distribution throughout the spine is unequal during activities that increase the load on the 
vertebrae.[10] During more strenuous daily activities (bending, twisting with weight in hands, lifting weights, pushing), compression loads are highest in common VF locations [10]. Interestingly, in subjects with only cVFs, we found that BA was only slightly decreased (- 15\%) compared to subjects without a VF. This suggests that these VFs are mainly associated with high compression loads related to such more strenuous activities that then exceeds the slightly decreased bone strength.

In contrast, in subjects with the combination of cVFs and lcVFs, BA was much lower (-32\%). In such cases, the load/ strength ratio of vertebrae is exceeded mainly in association with a lower BA. Even with daily activities of minor compression loads are than associated with VFs, at common and less common levels.

Interestingly, we also identified a group of subjects with only lcVFs. As less strenuous loads that occur in the less common fracture levels (due to, e.g. walking, getting up from a chair, tying shoes when sitting) still occur at normal magnitudes, these subjects could be more susceptible to VF only in these less common regions. One example is that shear load on vertebrae is higher in T6-T10 than in T11-T12 [27].

Thus, cVFs occurred in subjects with a higher BA and a lower load/BA ratio than in subjects with both cVFs and lcVFs, suggesting a combined role of BA and load in the location of VFs. However, the interpretation of these indirect calculations needs to be interpreted with caution and will need studies that combine the measurement of load and BA in the same subjects.

Briggs et al. [35] found that subjects with a VF had significantly greater normalised compression $(p=0.0008)$ and shear force $(p<0.0001)$ profiles and hypothesised that greater segmental flexion moments, compression forces and shear forces would exist in individuals with an osteoporotic vertebral fracture compared to those with osteoporosis and no history of vertebral fracture.

It is well known that assessment of VF status in addition to BMD provides relevant clinical information in predicting fracture risk [36]. The clinical implication of our finding is that subjects with lcVF have lower BA and may be at even higher fracture risk than subjects with only cVFs. The further useful clinical information is that BA alone cannot explain the locations of VFs, and those differences in load/BA ratio need further study to understand the heterogeneous locations of VFs. Our findings also contribute to further studies that investigate why most VFs are not accompanied by the acute signs and symptoms of an acute fracture and why most of the VFs occur subclinical, without overt acute trauma.

This study has several limitations.

First, this study was performed in smokers with and without COPD. Therefore, the results cannot be generalized to other populations, as smoking and COPD are independent risk factors for VFs and have different pathophysiology as compared to postmenopausal women and elderly [37, 38]. However, the peaks of prevalent VFs were similar as found in population studies in postmenopausal women and in men.

Second, we only evaluated BA non-fractured VFs, as BA can be increased following fracture healing with callus formation and thus not reflect its pre-fracture BA.

Third, we evaluated BA within the central region of the vertebrae. Intravertebral BA measured by QCT is significantly correlated with in vitro compressive strength of the vertebrae [39-41].

Fourth, we had no data on fall or trauma history, so we could not evaluate whether a VF was the result of a fall or other trauma or whether they occurred subclinical [42]. We neither have data on the level of physical activity.

Fifth, we did not evaluate adjacent intervertebral disc height and kyphosis angles that also influence how compressive forces are distributed over the vertebral body [15].

Sixth, the different CT scanners were not cross-calibrated, which may have influenced our results. Also differences between scanner manufacturers were not investigated for this study. However, scanners were regularly calibrated using industry and institutional standards. Although asynchronous calibration using a phantom or internal phantomless calibration is advocated when using different CT scans, in a review, it was cited that some have suggested using the CT values directly without any calibration to BMD [43].

Seventh, we did not measure lumbar spine vertebrae, as they are not included on chest CT scans.

Due to these limitations, further studies will be needed that integrate load and BA to explore the reasons of unequal distribution of VF in the spine.

In conclusion, the association between VFs and BA differs according to the location of VFs and ORs increases from subjects with cVFs only, to subjects with lcVFs and were the highest in subjects with cVFs and lcVFs combined, indicating that other factors than only BA play a role in the bimodal distribution of VFs. Prospective studies will be needed that examine the association between BA in non-fractured vertebrae at baseline and the incidence of new VFs according to their location.

Funding This work was financially supported by Stichting De Weijerhorst. This research was performed independently from funders.

\section{Compliance with ethical standards}

Conflicts of interest J.H.M. Driessen, M.J. van Dort, E.A.P.M. Romme, F.W.J.M. Smeenk and B. van Rietbergen declare that they have no conflict of interest.

E.F.M. Wouters reports board membership at Boehringer, grants and speaker fees from AstraZeneca, grants and speaker fees from GSK, speaker fees from Novartis and speaker fees from Chiesi, outside the submitted work.

J.P.W. van den Bergh reports grants from Eli Lilly, grants from Will Pharma and grants from Amgen, outside the submitted work. 
P. Geusens reports grants, speaker fees and advisory board from Amgen, grants from Pfizer, grants from MSD, grants from UCB, grants from Abbott, grants and speaker fees from Lilly, grants from BMS, grants from Novartis, grants from Roche and grants from Will Pharma, outside the submitted work.

Open Access This article is licensed under a Creative Commons Attribution-NonCommercial 4.0 International License, which permits any non-commercial use, sharing, adaptation, distribution and reproduction in any medium or format, as long as you give appropriate credit to the original author(s) and the source, provide a link to the Creative Commons licence, and indicate if changes were made. The images or other third party material in this article are included in the article's Creative Commons licence, unless indicated otherwise in a credit line to the material. If material is not included in the article's Creative Commons licence and your intended use is not permitted by statutory regulation or exceeds the permitted use, you will need to obtain permission directly from the copyright holder. To view a copy of this licence, visit http:// creativecommons.org/licenses/by-nc/4.0/.

\section{References}

1. Sambrook P, Cooper C (2006) Osteoporosis. Lancet 367(9527): 2010-2018

2. Cauley JA, Palermo L, Vogt M, Ensrud KE, Ewing S, Hochberg M, Nevitt MC, Black DM (2008) Prevalent vertebral fractures in black women and white women. J Bone Miner Res 23(9):1458-1467

3. van der Klift M, de Laet CEDH, McCloskey EV, Johnell O, Kanis JA, Hofman A, Pols HAP (2004) Risk factors for incident vertebral fractures in men and women: the Rotterdam Study. J Bone Miner Res 19(7):1172-1180

4. Bhambhani M, Crisp AJ, Compston JE (1992) Differential involvement of the dorsal and lumbar spine in osteoporosis. Ann Rheum Dis 51(9): 1069-1070

5. Oei L, Koromani F, Rivadeneira F, Zillikens MC, Oei EH (2016) Quantitative imaging methods in osteoporosis. Quant Imaging Med Surg 6(6):680-698

6. Melton LJ 3rd et al (1993) Prevalence and incidence of vertebral deformities. Osteoporos Int 3(3):113-119

7. Nevitt MC, Ross PD, Palermo L, Musliner T, Genant HK, Thompson DE (1999) Association of prevalent vertebral fractures, bone density, and alendronate treatment with incident vertebral fractures: effect of number and spinal location of fractures. The Fracture Intervention Trial Research Group. Bone 25(5):613-619

8. Anderson DE, Demissie S, Allaire BT, Bruno AG, Kopperdahl DL, Keaveny TM, Kiel DP, Bouxsein ML (2014) The associations between QCT-based vertebral bone measurements and prevalent vertebral fractures depend on the spinal locations of both bone measurement and fracture. Osteoporos Int 25(2):559-566

9. Geusens P, Kendler DL, Fahrleitner-Pammer A, López-Romero P, Marin F (2020) Distribution of prevalent and incident vertebral fractures and their association with bone mineral density in postmenopausal women in the teriparatide versus risedronate VERO Clinical Trial. Calcif Tissue Int 106(6):646-654

10. Bruno AG, Burkhart K, Allaire B, Anderson DE, Bouxsein ML (2017) Spinal loading patterns from biomechanical modeling explain the high incidence of vertebral fractures in the thoracolumbar region. J Bone Miner Res 32(6):1282-1290

11. Ignasiak D, Rüeger A, Sperr R, Ferguson SJ (2018) Thoracolumbar spine loading associated with kinematics of the young and the elderly during activities of daily living. J Biomech 70:175-184

12. Bachmann KN, Bruno AG, Bredella MA, Schorr M, Lawson EA, Gill CM, Singhal V, Meenaghan E, Gerweck AV, Eddy KT,
Ebrahimi S, Koman SL, Greenblatt JM, Keane RJ, Weigel T, Dechant E, Misra M, Klibanski A, Bouxsein ML, Miller KK (2016) Vertebral strength and estimated fracture risk across the BMI spectrum in women. J Bone Miner Res 31(2):281-288

13. Myers ER, Wilson SE (1997) Biomechanics of osteoporosis and vertebral fracture. Spine (Phila Pa 1976) 22(24 Suppl):25s-31s

14. Leucht P, Fischer K, Muhr G, Mueller EJ (2009) Epidemiology of traumatic spine fractures. Injury 40(2):166-172

15. Adams MA, Dolan P (2011) Biomechanics of vertebral compression fractures and clinical application. Arch Orthop Trauma Surg 131(12):1703-1710

16. Romme EA, Murchison JT, Phang KF, Jansen FH, Rutten EPA, Wouters EFM, Smeenk FWJM, van Beek EJR, MacNee W (2012) Bone attenuation on routine chest $\mathrm{CT}$ correlates with bone mineral density on DXA in patients with COPD. J Bone Miner Res 27(11): 2338-2343

17. van Dort MJ, Geusens P, Driessen JHM, Romme EAPM, Smeenk FWJM, Wouters EFM, van den Bergh JPW (2018) High imminent vertebral fracture risk in subjects with COPD with a prevalent or incident vertebral fracture. J Bone Miner Res 33(7):1233-1241

18. van Dort MJ et al (2018) Diagnosis of vertebral deformities on chest $\mathrm{CT}$ and DXA compared to routine lateral thoracic spine X-ray. Osteoporos Int 29:1285

19. Vestbo J, Anderson W, Coxson HO, Crim C, Dawber F, Edwards L, Hagan G, Knobil K, Lomas DA, MacNee W, Silverman EK, Tal-Singer R, ECLIPSE investigators (2008) Evaluation of COPD longitudinally to identify predictive surrogate end-points (ECLIPSE). Eur Respir J 31(4):869-873

20. Agusti A et al (2010) Characterisation of COPD heterogeneity in the ECLIPSE cohort. Respir Res 11:122

21. Hurst JR, Vestbo J, Anzueto A, Locantore N, Müllerova H, TalSinger R, Miller B, Lomas DA, Agusti A, Macnee W, Calverley P, Rennard S, Wouters EF, Wedzicha JA, Evaluation of COPD Longitudinally to Identify Predictive Surrogate Endpoints (ECLIPSE) Investigators (2010) Susceptibility to exacerbation in chronic obstructive pulmonary disease. N Engl J Med 363(12): $1128-1138$

22. Vestbo J, Hurd SS, Agustí AG, Jones PW, Vogelmeier C, Anzueto A, Barnes PJ, Fabbri LM, Martinez FJ, Nishimura M, Stockley RA, Sin DD, Rodriguez-Roisin R (2013) Global strategy for the diagnosis, management, and prevention of chronic obstructive pulmonary disease: GOLD executive summary. Am J Respir Crit Care Med 187(4):347-365

23. Brett A, Miller CG, Hayes CW, Krasnow J, Ozanian T, Abrams K, Block JE, van Kuijk C (2009) Development of a clinical workflow tool to enhance the detection of vertebral fractures: accuracy and precision evaluation. Spine 34(22):2437-2443

24. van der Velde R, Ozanian T, Dumitrescu B, Haslam J, Staal J, Brett A, van den Bergh J, Geusens P (2015) Performance of statistical models of shape and appearance for semiautomatic segmentations of spinal vertebrae T4-L4 on digitized vertebral fracture assessment images. Spine J 15(6):1248-1254

25. Genant HK et al (1993) Vertebral fracture assessment using a semiquantitative technique. J Bone Miner Res 8(9):1137-1148

26. Budoff MJ, Hamirani YS, Gao YL, Ismaeel H, Flores FR, Child J, Carson S, Nee JN, Mao S (2010) Measurement of thoracic bone mineral density with quantitative CT. Radiology 257(2):434-440

27. van Dort MJ et al (2019) Thoracic kyphosis on chest CT scans is associated with incident vertebral fractures in smokers. J Bone Miner Res 34:859

28. Keaveny TM, Bouxsein ML (2008) Theoretical implications of the biomechanical fracture threshold. J Bone Miner Res 23(10):15411547

29. Oei L, Koromani F, Breda SJ, Schousboe JT, Clark EM, van Meurs JBJ, Ikram MA, Waarsing JH, van Rooij FJA, Zillikens MC, Krestin GP, Oei EHG, Rivadeneira F (2018) Osteoporotic vertebral 
fracture prevalence varies widely between qualitative and quantitative radiological assessment methods: the Rotterdam Study. J Bone Miner Res 33(4):560-568

30. Van der Klift $\mathrm{M}$ et al (2002) The incidence of vertebral fractures in men and women: the Rotterdam Study. J Bone Miner Res 17(6): $1051-1056$

31. Sornay-Rendu E, Cabrera-Bravo JL, Boutroy S, Munoz F, Delmas PD (2009) Severity of vertebral fractures is associated with alterations of cortical architecture in postmenopausal women. J Bone Miner Res 24(4):737-743

32. Duan Y, Seeman E, Turner CH (2001) The biomechanical basis of vertebral body fragility in men and women. J Bone Miner Res 16(12):2276-2283

33. Romagnoli E, Carnevale V, Nofroni I, D’Erasmo E, Paglia F, de Geronimo S, Pepe J, Raejntroph N, Maranghi M, Minisola S (2004) Quality of life in ambulatory postmenopausal women: the impact of reduced bone mineral density and subclinical vertebral fractures. Osteoporos Int 15(12):975-980

34. Geusens P, Bours SPG, Wyers CE, van den Bergh JP (2019) Fracture liaison programs. Best Pract Res Clin Rheumatol 33(2): 278-289

35. Briggs AM, Wrigley TV, van Dieën JH, Phillips B, Lo SK, Greig AM, Bennell KL (2006) The effect of osteoporotic vertebral fracture on predicted spinal loads in vivo. Eur Spine J 15(12):17851795

36. Siris ES, Genant HK, Laster AJ, Chen P, Misurski DA, Krege JH (2007) Enhanced prediction of fracture risk combining vertebral fracture status and BMD. Osteoporos Int 18(6):761-770
37. da Rocha Lemos Costa TM et al (2018) Bone mineral density and vertebral fractures and their relationship with pulmonary dysfunction in patients with chronic obstructive pulmonary disease. Osteoporos Int 29(11):2537-2543

38. Thorin MH, Wihlborg A, Åkesson K, Gerdhem P (2016) Smoking, smoking cessation, and fracture risk in elderly women followed for 10 years. Osteoporos Int 27(1):249-255

39. Biggemann M, Hilweg D, Brinckmann P (1988) Prediction of the compressive strength of vertebral bodies of the lumbar spine by quantitative computed tomography. Skelet Radiol 17(4):264-269

40. Kopperdahl DL, Morgan EF, Keaveny TM (2002) Quantitative computed tomography estimates of the mechanical properties of human vertebral trabecular bone. J Orthop Res 20(4):801-805

41. Crawford RP, Cann CE, Keaveny TM (2003) Finite element models predict in vitro vertebral body compressive strength better than quantitative computed tomography. Bone 33(4):744-750

42. Jiang G, Luo J, Pollintine P, Dolan P, Adams MA, Eastell R (2010) Vertebral fractures in the elderly may not always be "osteoporotic". Bone 47(1):111-116

43. Engelke K, Libanati C, Fuerst T, Zysset P, Genant HK (2013) Advanced CT based in vivo methods for the assessment of bone density, structure, and strength. Curr Osteoporos Rep 11(3):246255

Publisher's note Springer Nature remains neutral with regard to jurisdictional claims in published maps and institutional affiliations. 